\title{
Expanding living kidney donor criteria with ex-vivo surgery for renal anomalies
}

\author{
Thomas B. McGregor, MD;' Christie Rampersad, MD;' Premal Patel, MD'
}

ISection of Urology; ${ }^{2}$ College of Medicine; University of Manitoba, Winnipeg, MB, Canada

Cite as: Can Urol Assoc J 2016;10(9-10):301-5. http://dx.doi.org/10.5489/cuai.3841

\section{Abstract}

Introduction: Renal transplantation remains the gold standard treatment for end-stage renal disease, with living donor kidneys providing the best outcomes in terms of allograft survival. As the number of patients on the waitlist continues to grow, solutions to expand the donor pool are ongoing. A paradigm shift in the eligibility of donors with renal anomalies has been looked at as a potential source to expand the living donor pool. We sought to determine how many patients presented with anatomic renal anomalies at our transplant centre and describe the ex-vivo surgical techniques used to render these kidneys suitable for transplantation.

Methods: A retrospective review was performed of all patients referred for surgical suitability to undergo laparoscopic donor nephrectomy between January 2011 and January 2015. Patient charts were analyzed for demographic information, perioperative variables, urological histories, and postoperative outcomes.

Results: 96 referrals were identified, of which 81 patients underwent laparoscopic donor nephrectomy. Of these patients, 11 $(13.6 \%)$ were identified as having a renal anomaly that could potentially exclude them from the donation process. These anomalies included five patients with unilateral nephrolithiasis, four patients with large renal cysts ( $>4 \mathrm{~cm}$ diameter), one patient with an angiomyolipoma (AML) and one patient with a calyceal diverticulum filled with stones. A description of the ex-vivo surgical techniques used to correct these renal anomalies is provided.

Conclusions: We have shown here that ex-vivo surgical techniques can safely and effectively help correct some of these renal anomalies to render these kidneys transplantable, helping to expand the living donor pool.

\section{Introduction}

Renal transplantation remains the gold standard treatment for end-stage renal disease (ESRD); ${ }^{1}$ however, the number of patients on the transplant waitlist continues to expand, with 5333 newly diagnosed patients with ESRD diagnosed in 2013; this added to the pre-existing pool of 24114 patients living with this condition on dialysis. Since 1994, the preva- lence rate for patients being treated by dialysis has increased by $123 \% .^{2}$ Despite this, the number of available donors from all sources has come to a plateau and the search for new and innovative ways to expand the donor pool is ongoing. In Canada, approximately 1200-1300 kidneys are transplanted each year, with $55 \%$ of these transplants arising from the deceased donor pool. ${ }^{2}$ Current efforts to expand the deceased donor pool include use of marginal deceased donors, namely expanded criteria donors, as well as donation after cardiocirculatory death. ${ }^{3}$ These efforts have been fairly successful, with deceased donor numbers in both North America and Europe having risen over the past 10 years.

Nonetheless, living kidney donation remains the pillar of all renal transplant programs, as living donors provide kidneys with the best outcomes in terms of allograft survival and delayed graft function rates. ${ }^{4}$ Several efforts have been instituted over the years to help improve the rate of living kidney donation. Firstly, with the introduction of minimally invasive kidney surgery, laparoscopic techniques have attracted more individuals to consider donation, mainly due to the decreased morbidity and shorter recovery time of this procedure. 5,6 Secondly, the advent of living-donor pairedexchange programs (LDPE) has helped to both increase living donor numbers and provide kidneys for recipients who are highly sensitized. ${ }^{7,8}$ Along with the LDPE, incorporation of non-directed altruistic donors has also helped improve donor numbers by initiating several LDPE chains. ${ }^{7,8}$

A final way to increase the living donor pool is to use marginal-living donors. Although there is no established definition of a marginal-living donor, these donors can include elderly, obese, or donors with renal anomalies that would typically exclude them from kidney donation. Although limited literature exists, kidneys with renal anomalies have been used for transplant with excellent outcomes. Some of these anomalies include nephrolithiasis, ureteropelvic junction obstruction, and kidneys with a small renal mass. ${ }^{9-12}$

Herein, we report on four separate renal anomalies that have arisen on multiple occasions at our transplant program. Each of these renal anomalies would typically exclude donors 
from the donation process. We describe the workup, donor surgery, and ex-vivo techniques used to treat these renal anomalies, rendering the kidneys acceptable for transplantation.

\section{Methods}

At our centre, all candidates for living kidney donation are originally worked up by the transplant-nephrology team and all medical contraindications to donation are eliminated at this point. Once the patient is cleared from both a medical and immunological standpoint, they are referred to the surgical-transplant team to be evaluated for surgical suitability to undergo laparoscopic donor nephrectomy. At this point, donors are screened closely for any surgical contraindications to kidney donation, including anatomic and renal anomalies.

Following approval of the ethics review board at our institution, we performed a review of all referrals to the surgicaltransplant team for suitability to undergo laparoscopic donor nephrectomy. All referrals from January 2011 to January 2015 were reviewed. We analyzed the charts for patient demographics, preoperative variables, operative details, and postoperative outcomes up to one year of followup. We focused specifically on the urological histories of all donors and identified renal anomalies from both the history and imaging (ultrasound and computed tomography [CT]) that would normally exclude candidates from the donation process.

\section{Results}

A total of 96 consecutive referrals were identified. Of these, nine donors were eliminated after identification of an absolute surgical contraindication to kidney donation. These absolute surgical contraindications included five patients with bilateral renal calculi, two patients with renal artery stenosis, one patient with evidence of renal atrophy and scarring, and one patient with evidence of fibromuscular dysplasia (Fig. 1). Eighty-seven of these referrals were approved for laparoscopic kidney donation and of these, 81 went on to donate a kidney.

Of the 81 patients that were both approved and then successfully underwent laparoscopic kidney donation, 11 patients were identified that had renal anomalies that required ex-vivo surgical correction prior to transplantation (Table 1). These renal anomalies could be broken down into four categories: five patients with unilateral nephrolithiasis, four patients with large renal cysts (defined as $>4 \mathrm{~cm}$ diameter), one patient with an angiomyolipoma (AML), and one patient with a calyceal diverticulum filled with stones.

Each of these renal anomalies were corrected or treated with ex-vivo surgical techniques that rendered the kidney acceptable for transplantation. Below, we outline the ex-vivo techniques used for these four categories of renal anomalies

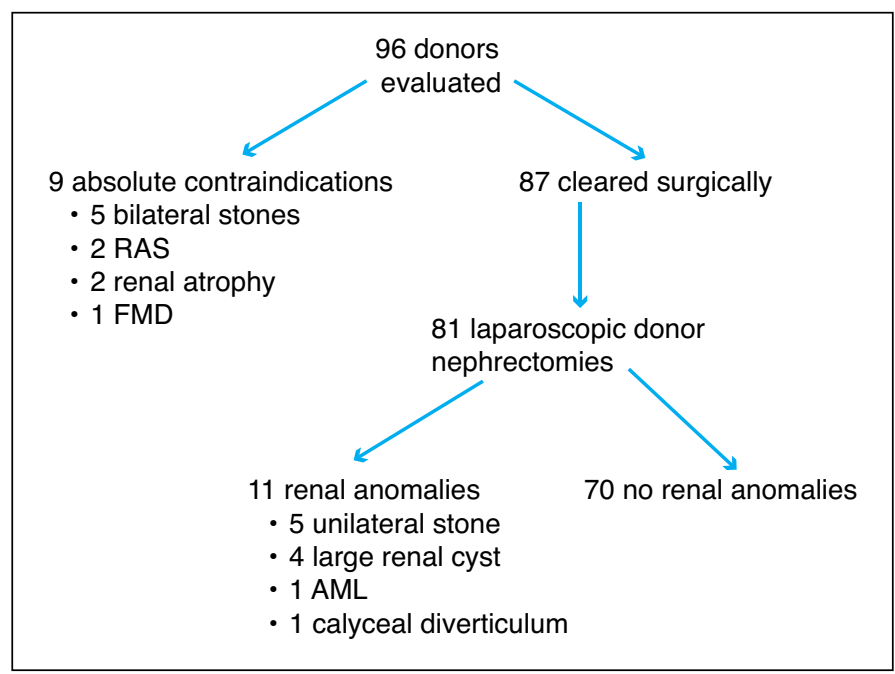

Fig. 1. Breakdown of living donor candidates evaluated for surgery. AML: angiomyolipoma; FMD: fibromuscular dysplasia; RAS: renal artery stenosis.

identified in our series, as well as outcomes for the donor and recipient up to one year of followup.

\section{Unilateral nephrolithiasis}

Five patients with incidentally discovered unilateral nephrolithiasis were identified. All donors denied a history of renal colic or prior stone disease. Metabolic stone disease workup in all cases was negative. All cases represented a solitary stone with an average size of $3 \mathrm{~mm}$ (range $2-5 \mathrm{~mm}$ ).

Following laparoscopic donor nephrectomy, perfusate flush, and cooling of the kidney, the ureter was spatulated distally to facilitate passage of a flexible ureteroscope. Systematic inspection the renal pelvis and all calyces were performed (Fig. 1). In each case, the stone was small enough to be removed with a nitinol basket. No form of stone breakage was required. There were no immediate complications or ureteric injuries related to ex-vivo ureteroscopy. Following ex-vivo

\begin{tabular}{|c|c|c|c|}
\hline Outcome & $\begin{array}{c}\text { No renal } \\
\text { anomalies } \\
\mathrm{n}=70(86 \%)\end{array}$ & $\begin{array}{c}\text { Renal } \\
\text { anomalies } \\
n=11(14 \%)\end{array}$ & p value \\
\hline Age (years)* & 47 & 45.5 & 0.91 \\
\hline Body mass index $\left(\mathrm{kg} / \mathrm{m}^{2}\right)^{*}$ & 26.6 & 24.9 & 0.06 \\
\hline $\begin{array}{l}\text { Preoperative creatine } \\
\text { (umol/L)* }\end{array}$ & 71.1 & 75.2 & 0.28 \\
\hline Male:Female & $29: 41$ & $7: 4$ & 0.20 \\
\hline Right:Left & $18: 52$ & $3: 8$ & 1.00 \\
\hline Cold ischemic time $(\min )^{*}$ & 45.4 & 53.6 & 0.03 \\
\hline Estimated blood loss $(\mathrm{mL})^{*}$ & 306 & 359 & 0.43 \\
\hline Length of stay (days)* & 2.3 & 2.5 & 0.53 \\
\hline
\end{tabular}


ureteroscopy and basket stone extraction, each kidney was then transplanted into the respective recipient. Recipient surgery was uneventful in each case and ureters were stented as is usual for all kidney transplants at our centre.

Ongoing surveillance imaging with plain film X-ray of the contralateral donor kidney was recommended on a 1-2 yearly basis going forward. Donors were also counselled towards dietary modifications and fluid intake to help prevent stone formation in the future. Recipients underwent similar dietary counselling and surveillance imaging of the renal allograft with plain film X-ray planned on an annual basis. At one year of followup, no recurrent stone disease was evident in the five donors or recipients.

\section{Large renal cysts}

Four patients were identified with large renal cysts. These cysts were classified as Bosniak type 1 as they had no evidence of septations, wall calcifications, or wall enhancement (Fig. 2). All contralateral renal units in these donors were normal.

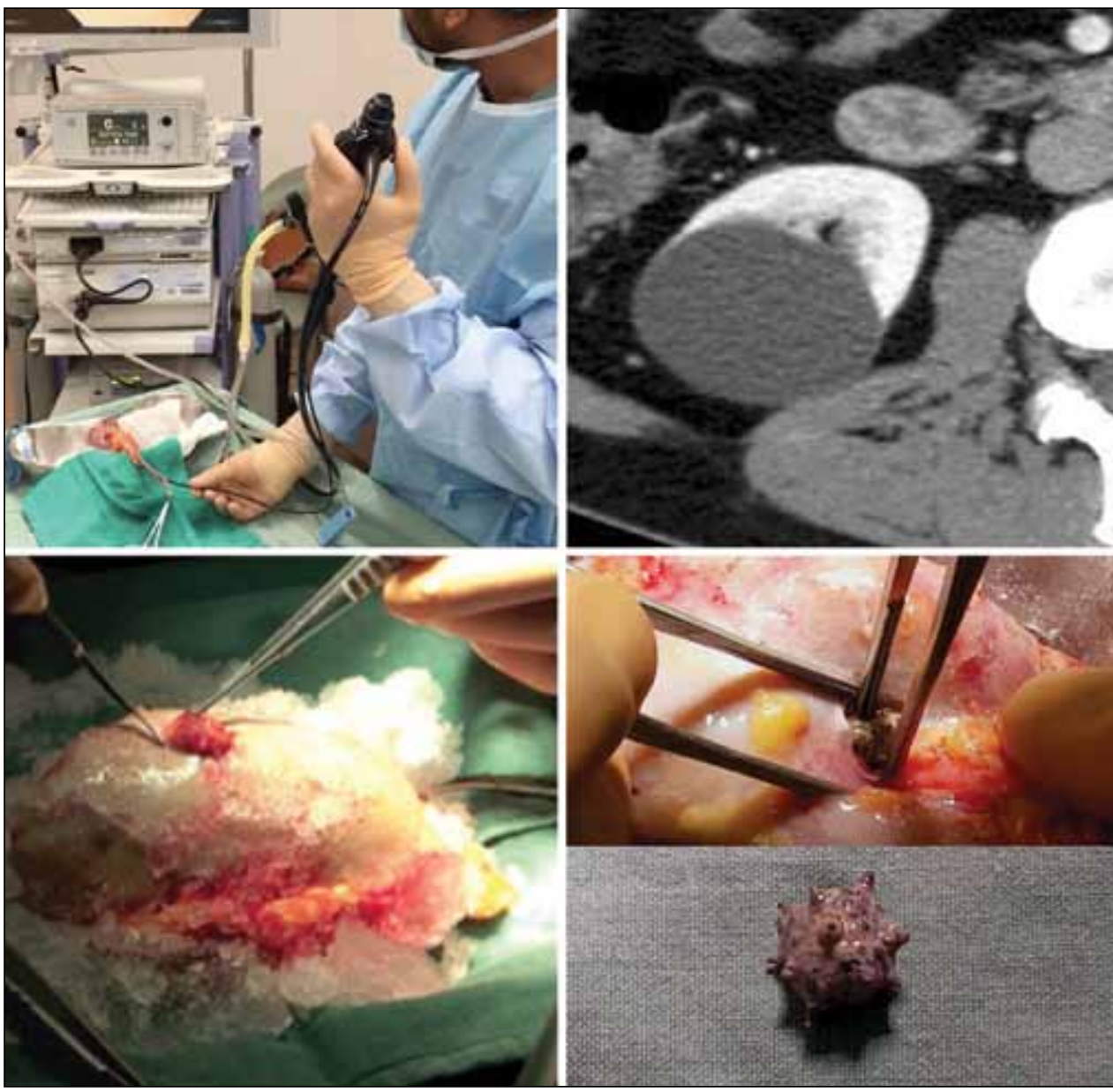

Fig. 2. Ex-vivo ureteroscopy for renal calculi (top left); computed tomography (CT) image of large right renal cyst (top right); ex-vivo partial nephrectomy for angiomyolipoma (bottom left); ex-vivo nephrolithotomy for stone removal from a calyceal diverticulum (bottom right).
In each case, the donor underwent a routine laparoscopic donor nephrectomy followed by ex-vivo deroofing of these cysts using metzenbaum scissors. This was followed by argon beam ablation of the inner cyst lining. Each kidney was then transplanted into the respective recipient. There was no significant bleeding from the cyst rim or parenchyma in each case that could not be controlled with pinpoint cautery. Postoperative course for the recipients in all four cases was uneventful, with no evidence of bleeding.

Followup consisted of an ultrasound of the donor's contralateral kidney at one year, which was negative in each case. Seeing as the renal cysts were thought to be sporadic, no routine donor imaging followup was planned thereafter. Recipient followup consisted of an allograft ultrasound at six months, which was negative for cyst recurrence in each case, with ongoing plans for a yearly ultrasound thereafter.

\section{Angiomyolipoma}

One patient with an incidentally discovered solitary AML was identified. The patient had no history or clinical features consistent with a hereditary form of AML. The AML had a diameter of $2.2 \mathrm{~cm}$ and had the pathognomonic findings of macroscopic fat on CT scanning.

This patient underwent a routine laparoscopic donor nephrectomy followed by ex-vivo partial nephrectomy and renorrhaphy. At the backtable sharp dissection of perinephric fat off the kidney was followed by dissection with enucleation of the AML from within the parenchyma of the kidney (Fig. 2). The mass was sent for frozen and confirmed to be a benign AML. The deep margin of the defect was then oversewn with a running 4-0 PDS suture to seal off any potential vascular defects. The cavity was then filled with a small amount of Floseal $^{\mathrm{TM}}$ and a Surgicel ${ }^{\mathrm{TM}}$ bolster. The parenchyma was then closed overtop with interrupted 2-0 PDS suture. The kidney was then successfully transplanted into the recipient with no bleeding or urine leak occurring during the perioperative period.

At one year followup, neither the donor nor recipient had any evidence of recurrent $A M L$ formation. Seeing as the AML appears to be 
sporadic, chances of developing a second lesion are low. However, plans are in place for ultrasound surveillance of both the donor contralateral kidney and the recipient renal allograft approximately every two years.

\section{Calyceal diverticulum}

One patient with a solitary calyceal diverticulum was identified. The diverticulum was found incidentally on imaging, measuring $2.3 \mathrm{~cm}$ in diameter and containing a $1.2 \mathrm{~cm}$ stone. The kidney otherwise had no other abnormalities and the contralateral kidney was completely normal. The patient denied a history of renal colic, infection or hematuria secondary to the calyceal diverticulum and stone.

This patient underwent a routine laparoscopic donor nephrectomy followed by ex-vivo ureteroscopy (as described above), open nephrolithotomy, and ablation of the calyceal diverticulum. The ureteroscopy confirmed no obvious connection between the collecting system and the calyceal diverticulum. Ultrasound was then used to identify the diverticulum and stone, allowing passage of a 25 -gauge needle onto the stone. A scalpel was then used to cut a $2 \mathrm{~cm}$ defect into the diverticulum. The stone was easily identified and removed with forceps (Fig. 2). The floor of the diverticulum was then oversewn with 5-0 PDS suture to close off any potential opening with the collecting system. The walls of the diverticulum were then ablated with argon beam followed by closure of the parenchymal defect with interrupted 2-0 PDS suture. Successful transplantation of the kidney took place with no findings of bleeding or urine leak from the resection site postoperatively.

At one year followup, neither the donor nor the recipient had any evidence of recurrent stone formation. The recipient was followed with an ultrasound every six months, with plans for a yearly ultrasound thereafter. The donor had a kidney-ureter-bladder (KUB) X-ray performed at one year showing no evidence of stones or calcifications over the contralateral kidney. With a negative metabolic stone workup and the thought that the original stone was formed due to an anatomic abnormality (calyceal diverticulum), no routine imaging of the donor is planned thereafter.

All 11 of these kidneys were transplanted with no intraoperative complications. Postoperatively, there were no significant complications other than one day of limited hematuria in one recipient and an episode of urinary retention in another following catheter removal on Day 4 (Table 2). Also, there were no episodes of delayed graft function. At one year, all allografts were functioning well with excellent creatinine values.

\section{Discussion}

The 11 cases presented above reveal how ex-vivo techniques can help correct anatomic renal anomalies, rendering

\begin{tabular}{|c|c|c|c|c|}
\hline Recipient & DGF & Complications & $\begin{array}{l}\text { Length } \\
\text { of stay } \\
\text { (days) }\end{array}$ & $\begin{array}{l}\text { 1-year } \\
\text { creatine } \\
\text { (umol/L) }\end{array}$ \\
\hline \#1 renal stone & No & Nil & 5 & 98 \\
\hline \#2 renal stone & No & Nil & 5 & 109 \\
\hline \#3 renal stone & No & Hematuria $x 1$ day & 7 & 102 \\
\hline \#4 renal stone & No & Nil & 6 & 87 \\
\hline \#5 renal stone & No & Nil & 6 & 153 \\
\hline \#6 renal cyst & No & $\begin{array}{l}\text { Urinary tract } \\
\text { infection }\end{array}$ & 7 & 140 \\
\hline \#7 renal cyst & No & Nil & 5 & 95 \\
\hline \#8 renal cyst & No & Urinary retention & 7 & 110 \\
\hline \#9 renal cyst & No & Nil & 5 & 84 \\
\hline \#10 AML & No & Nil & 6 & 107 \\
\hline $\begin{array}{l}\text { \#11 calyceal } \\
\text { diverticulum }\end{array}$ & No & Nil & 6 & 130 \\
\hline
\end{tabular}

kidneys that would normally not be considered for donation to be transplantable. Although this alone will not cure the kidney shortage, it certainly will help to at least partially alleviate the problem. In our series alone, 11 of 81 donors were found to have renal anomalies. This represents $14 \%$ of our living donor pool that may have been potentially turned down. When you extrapolate this number nationally to all transplant programs, these numbers add up and you can see the big impact this could have in helping to increase the number of living donors used nationwide.

Nephrolithiasis was historically considered a contraindication to living kidney donation due to the concerns regarding recurrent stone formation in the donor's solitary kidney and concerns of graft loss in the recipient. ${ }^{9}$ However, attitudes towards this have changed in individuals with no prior stone history and those with a negative metabolic workup. Our results corroborate the findings of several studies that have demonstrated the safety and ease of performing ex-vivo ureteroscopy prior to renal implantation. . $^{10,13,14}$

With regards to small renal masses, one patient with an incidentally discovered $2 \mathrm{~cm}$ AML underwent ex-vivo partial nephrectomy and renorrhaphy. Ex-vivo partial nephrectomy for incidentally discovered renal masses with subsequent renal implantation has been described previously, with several reports demonstrating its applicability to small renal cell carcinomas. ${ }^{11,15-17}$ In a study of 14 patients who underwent renal implantation after ex-vivo partial nephrectomy for small renal masses (median size $2 \mathrm{~cm}$ ), there was no evidence of recurrence after a mean followup of 69 months. ${ }^{16}$ Other renal masses, such as cysts, can be managed quite easily with deroofing and argon beam ablation of the inner cyst lining. A retrospective analysis of 25 patients with renal transplantation from living marginal donors with renal cysts showed no cyst-related complications or graft dysfunction in these recipients. ${ }^{18}$ 
Finally, these ex-vivo surgical techniques represent skills that all urologist possess. In fact, the ex-vivo nature of the procedure, bloodless field, and lack of time restraint from warm ischemic time arguably makes these ex-vivo procedures more straightforward and safe. ${ }^{19}$ In our series, none of the transplants following our 11 ex-vivo procedures resulted in extraneous bleeding, urine leak, or unfavourable outcome. There was also no evidence of delayed graft function and allograft function at one year was excellent.

Our results must be taken within the context of the study limitations. This was a retrospective analysis with limitations inherent to this design. Furthermore, these results represent the experience of a single centre and other transplant centres may have more strict exclusion criteria for living kidney donors in regards to renal anomalies. However, this study does present a real-life look at four consecutive years of living kidney donors and the types of renal anomalies that can present themselves during workup.

\section{Conclusion}

With the number or patients on the transplant waitlist continuing to grow, efforts to expand both the deceased and living donor pools is ongoing. Despite successful efforts, such as LDPE, to expand living donor numbers worldwide, there are still donors who are turned down each year due to findings of renal anomalies on the donor workup. We have shown here that ex-vivo surgical techniques can safely and effectively help correct some of these renal anomalies to render these kidneys transplantable, helping to expand the living donor pool.

Competing interests: The authors report no competing personal or financial interests.

This paper has been peer-reviewed.

\section{References}

1. McGregor T, Biazevic J, Patel P, et al. Changing of the guard? A glance at the surgical representation in the Canadian renal transplantation community. Can Urol Assoc J 2016;10:E7-11. http://dx.doi. org/10.5489/cuaj.3256

2. Canadian Institute for Health Information. Canadian Organ Replacement Register Annual Report: Treatment of end-stage organ failure in Canada, 2004 to 2013. Ottawa, ON: CIHI:2015. Available at https://secure. cihi.ca/free_products/2015_CORR_AnnualReport_ENweb.pdf. Accessed March 28, 2016.
3. Canadian Institute for Health Information. Deceased Organ Donor Potential in Canada. Ottawa, ON: CIHI:2014. Available at https://www.cihi.ca/web/resource/en/organdonorpotential_2014_en.pdf. Accessed March 30, 2016.

4. Naderi GH, Mehraban D, Kazemeyni SM, et al. Living or deceased donor kidney transplantation: A comparison of results and survival rates among Iranian patients. Transplant Proc 2009;41:2772-4. http://dx.doi.org/10.1016/i.transproceed.2009.07.041

5. Pradel FG, Mullins CD, Bartlett ST. Exploring donors' and recipients' attitudes about living donor kidney transplantation. Prog Transplant 2003;13:203-10. http://dx.doi.org/10.7182/ prtt.13.3.27710581808643g8

6. Rampersad C, Patel P, McGregor T, et al. Back-to-back comparison of mini-open vs. laparoscopic technique for living kidney donation. Can Urol Assoc J 2016;10(7-8):253-7. http://dx.doi.org/10.5489/ cuai.3725

7. Ferrari P, de Klerk M. Paired kidney donations to expand the living donor pool. J Nephrol 2009;22:699707.

8. Cole EH, Nickerson P, Campbell P, et al. The Canadian kidney paired donation program: A national program to increase living donor transplantation. Transplantation 2015;99:985-90. http://dx.doi.org/10.1097/ TP.0000000000000455

9. Sener A, Uberoi V, Bartlett ST, et al. Living-donor renal transplantation of grafts with incidental renal masses after ex-vivo partial nephrectomy. BJU Int 2009;104:1655-60. http://dx.doi.org/10.1111/i.1464410X.2009.08681.x

10. Bozkurt B, Kllıc M. Marginal donors in renal transplantation. Transplant Proc 2015;47:1273-81. http://dx.doi.org/10.1016/i.transproceed.2015.04.006

11. Olsburgh J, Thomas K, Wong K, et al. Incidental renal stones in potential live kidney donors: Prevalence, assessment, and donation, including role of ex-vivo ureteroscopy. BJU Int 2013;111:784-92. http:// dx.doi.org/10.1111/i.1464-410X.2012.11572.x

12. Lugo-Baruqui $A$, Guerra $G$, Arocha $A$, et al. Use of kidneys with small renal tumours for transplantation. Curr Urol Rep 2016;17:3. http://dx.doi.org/10.1007/s1 1934-015-0557-z

13. Rizkala E, Coleman $S$, Tran C, et al. Stone disease in living-related renal donors: Long-term outcomes for transplant donors and recipients. J Endourol 2013;27:1520-4. http://dx.doi.org/10.1089/ end.2013.0203

14. Schade GR, Wolf IS, Faerber GJ. Ex-vivo ureteroscopy at the time of live donor nephrectomy. J Endourol 2011;25:1405-9. http://dx.doi.org/10.1089/end.2010.0627

15. Nicol DL, Preston JM, Wall DR, et al. Kidneys from patients with small renal tumours: A novel source of kidneys for transplantation. BJU Int 2008;102:188-92. http://dx.doi.org/10.1111/j.1464410X.2008.07562.x

16. Buell JF, Hanaway MJ, Thomas $M$, et al. Donor kidneys with small renal cell cancers: Can they be transplanted? Transplant Proc 2005;37:581-2. http://dx.doi.org/10.1016/.t.transproceed.2004.12.118

17. Ogawa Y, Kojima K, Mannami R, et al. Transplantation of restored kidneys from unrelated donors after resection of renal cell carcinoma: Results from 10 patients. Transplant Proc 2015:47:1711-9. hitp://dx.doi.org/10.1016/i.transproceed.2015.06.030

18. Grotemeyer D, Voiculescu A, Iskandar $F$, et al. Renal cysts in living donor kidney transplantation: Longterm followup in 25 patients. Transplant Proc 2005;41:4047-51. http://dx.doi.org/10.1016/i. transproceed.2009.09.077

19. Nayak JG, Koulack J, McGregor TB. Laparoscopic nephrectomy, ex-vivo partial nephrectomy, and autotransplantation for the treatment of complex renal masses. Case Rep Urol 2014;2014:354104.

Correspondence: Dr. Thomas McGregor, Department of Urology, Queen's University, Kingston, ON, Canada; mcgregot@KGH.KARI.NET 